\title{
Pneumoperitoneum in a patient with Larsen syndrome
}

\author{
Satoki Inoue $\cdot$ Yasumitsu Nomura \\ Masahiko Kawaguchi
}

Received: 18 September 2013/Accepted: 21 October 2013/Published online: 9 November 2013

(C) Japanese Society of Anesthesiologists 2013

Keywords Pneumoperitoneum · Larsen syndrome · Mechanical ventilation

To the Editor:

Larsen syndrome is a congenital disorder, which consists of a skeletal dysplasia with multiple joint dislocations and a characteristic face [1]. The basis of the abnormalities is considered to be a generalized mesenchymal disorder involving connective tissues [2]. We describe a patient with Larsen syndrome who suffered from pneumoperitoneum during mechanical ventilation.

A 16-year-old boy with Larsen syndrome was admitted because of respiratory failure. Immediately after admission, his airway was established using a cuffed $6.0-\mathrm{mm}$ tracheal tube without a Murphy eye. His chest X-ray showed severe scoliosis and implied a possibility of endobronchial shift or carinal impingement of the tracheal tube. His deformity allowed only a margin of $1.0-0.5 \mathrm{~cm}$ between the tube end and the carina. Expiratory tidal volumes depended on patient positioning because of severe scoliosis. On the next day, his abdomen became tense and distended. A chest-abdominal X-ray revealed free air under the diaphragm and subcutaneous emphysema. In addition, carinal impingement of the tracheal tube was confirmed (Supplementary Fig. 1). A chest-abdominal CT showed free peritoneal air and emphysema in the anterior

Electronic supplementary material The online version of this article (doi:10.1007/s00540-013-1742-1) contains supplementary material, which is available to authorized users.

S. Inoue $(\bowtie) \cdot$ Y. Nomura $\cdot$ M. Kawaguchi

Division of Intensive Care, Nara Medical University,

840 Shijo-cho, Kashihara, Nara 634-8522, Japan

e-mail: seninoue@naramed-u.ac.jp abdominal wall (Supplementary Fig. 2). Pneumomediastinum and massive subcutaneous emphysema were confirmed (Supplementary Fig. 3). We attempted to correct the position of the tracheal tube, which impinged on the carina. With careful management regarding the tube position, his respiratory status finally improved.

The air leak was probably derived from a micro-tear of the trachea, because carinal impingement of the tracheal tube was repeatedly observed. The background status, which in this case means weakness of connective tissues due to Larsen syndrome, seemed to predispose to the air leak. Air may leak into the mediastinum and then into the retroperitoneal space and the peritoneum by the diaphragmatic openings [3]. After correction of the tube position, positive pressure was effectively delivered to alveoli instead of directly to the tracheal wall, which appeared to decrease the air leak and free peritoneal air.

Conflict of interest There is no conflict of interest.

\section{References}

1. Larsen LJ, Schottstaedt ER, Bost FC. Multiple congenital dislocations associated with characteristic facial abnormality. J Pediatr. 1950;37:574-81.

2. Kiel EA, Frias JL, Victorica BE. Cardiovascular manifestations in the Larsen syndrome. Pediatrics. 1983;71:942-6.

3. Addison NV, Broughton AC. Tension pneumoperitoneum: a report of 4 cases. Br J Surg. 1976;63:877-80. 